\begin{tabular}{|c|l|}
\hline Title & Energy renormalization of exciton complexes in GaA s quantum dots \\
\hline Author(s) & $\begin{array}{l}\text { A bbarchi, Marco; Kuroda, Takashi; Mano, Takaaki; Sakoda, Kazuaki; Mastrandrea, Carmine A .; V inattieri, A nna; } \\
\text { Gurioli, Massimo; Tsuchiya, Takuma }\end{array}$ \\
\hline Citation & $\begin{array}{l}\text { Physical Review B, 82(20), 201301 } \\
\text { https://doi.org/_0.1103/PhysRevB.82.201301 }\end{array}$ \\
\hline Issue Date & 2010-11-15 \\
\hline Doc URL & http://hdl.handle.net/2115/44389 \\
\hline Rights & @2010 The A merican Physical Society \\
\hline Type & article \\
\hline File Information & PRB82-20_201301.pdf \\
\hline
\end{tabular}

Instructions for use 


\title{
Energy renormalization of exciton complexes in GaAs quantum dots
}

\author{
Marco Abbarchi, ${ }^{1, *}$ Takashi Kuroda, ${ }^{1, \dagger}$ Takaaki Mano, ${ }^{1}$ Kazuaki Sakoda, ${ }^{1}$ Carmine A. Mastrandrea, ${ }^{2}$ Anna Vinattieri, ${ }^{2}$ \\ Massimo Gurioli, ${ }^{2}$ and Takuma Tsuchiya ${ }^{3}$ \\ ${ }^{1}$ National Institute for Materials Science, 1-1 Namiki, Tsukuba 305-0044, Japan \\ ${ }^{2}$ LENS, Dipartimento di Fisica, Università di Firenze, Via Sansone 1, 50019 Sesto Fiorentino, Italy \\ ${ }^{3}$ Division of Applied Physics, Hokkaido University, Sapporo 060-8628, Japan
}

(Received 5 July 2010; revised manuscript received 4 September 2010; published 4 November 2010)

\begin{abstract}
Using GaAs/AlGaAs self-assembled quantum dots we study the few-particle dynamics of fully confined systems, which is associated with purely Coulomb interaction and is not affected by the internal strain field. Systematic evolution in the binding energies of positive and negative trions, as well as biexcitons, with dot size is interpreted in terms of a balance between the Hartree mean-field corrections on single-particle states and the interparticle correlations which lead to a nonseparable dynamics. The experimental behaviors are well reproduced by exact many-body calculations within the framework of the quantum Monte Carlo approach.
\end{abstract}

DOI: 10.1103/PhysRevB.82.201301

Excitons $(\mathrm{X})$ and their multi-particles complexes, such as negative and positive trions $\left(\mathrm{X}^{-}\right.$and $\mathrm{X}^{+}$, respectively) as well as biexcitons (XX), are prototypical few-body systems realized in a solid. Their simplest atomic counterparts, such as $\mathrm{H}^{-}$, which have been studied since the earliest days of quantum mechanics, are regarded as the system of choice for studying the intricacies of few-body dynamics. ${ }^{1}$ Particles in $\mathrm{H}^{-}$(two electrons and one proton) are bound due to a delicate balance between the attractive and repulsive interactions, and the relatively strong correlations reflecting low kinetic energies involved. Consequently, the dissociation energy of $\mathrm{H}^{-}$is only about $0.75 \mathrm{eV}$, much smaller than the ionization energy of the Hydrogen atom $(13.6 \mathrm{eV})$.

Quantum heterostructures, which accommodate charged particles in nanoscopic space, allow us to investigate the impact of dimensionality and localization on the few-particle dynamics. For quantum wells (QWs) in the strong confinement limit, the $\mathrm{X}$ binding energy (BE) would be enhanced by a factor of four compared to that in the three-dimensional system. The two-dimensional enhancement in the BE of XX (Refs. 2 and 3) as well as $\mathrm{X}^{ \pm}$(Refs. 4 and 5) has been extensively studied. ${ }^{6-8}$

Few-particle BEs in fully confined systems have been precisely determined by single quantum-dot (QD) spectroscopy experiments. Peculiar features reported so far show that for a common class of QDs the $\mathrm{BE}$ of $\mathrm{X}^{-}$is positive, ${ }^{9}$ while that of $\mathrm{X}^{+}$is negative. ${ }^{10}$ This is in stark contrast to higher dimensionality systems, where the bound states exist for both $\mathrm{X}^{-}$ and $\mathrm{X}^{+}$in QWs, as well as for $\mathrm{H}^{-}$and $\mathrm{H}_{2}^{+}$in nature. Moreover they show that the BE of $\mathrm{XX}$ becomes negative for sufficiently small QDs. ${ }^{11}$ Unbound XX is frequently observed in nitride polar QDs, ${ }^{12}$ and, recently, in nonpolar QDs under in-plane strain. ${ }^{13}$ These results suggest that fewparticle states are crucially influenced by the internal (piezo or spontaneous) electric field, which can be as high as the Coulomb field between charged particles.

Various attempts using strained InGaAs QD systems have also shown that few-particle spectra depend heavily on the microscopic shape of each dot. Thus, in experiment, the size dependence of few-carrier levels has normally been observed with large scattering in data. This masked the intrinsic size dependence, and, in theory, qualitatively different size dependence was predicted depending on the QD shape. ${ }^{11}$ The
PACS number(s): 78.67.Hc, 73.21.La, 78.55.Cr

effect of quantum confinement on exciton complexes, associated with pure Coulomb interaction, still remains an open point.

It is the purpose of this Rapid Communication to study the quantum-confinement effect on exciton complexes in strain-free QD systems, thus ideally avoiding the influence of built-in electric fields. Using lattice-matched GaAs/AlGaAs QDs we observed the systematic evolution in $\mathrm{XX}$ and $\mathrm{X}^{ \pm}$ energies as a function of QD size, revealing a transition from the bound state to the unbound state for $\mathrm{X}^{+}$, which accounts for contradictory findings for the $\mathrm{X}^{+}$energies in QWs and QDs. The experimental behavior was in quantitative agreement with the few-particle energies evaluated by quantum Monte Carlo (QMC) calculations. Our results offer a global understanding in the quantum-size effect on few-particle states, which has been missed in previous works using strained QD systems.

GaAs QDs were grown on the (311) $\mathrm{A} \mathrm{Al}_{0.24} \mathrm{Ga}_{0.76} \mathrm{As}$ (sample A) and the (100) $\mathrm{Al}_{03} \mathrm{Ga}_{07} \mathrm{As}$ (sample B) by droplet epitaxy. ${ }^{14-16}$ The lens-shaped QDs in sample A (B) are characterized by $35(15) \mathrm{nm}$ in base diameter and 4 (6) $\mathrm{nm}$ in height. Single QD photoluminescence (PL) was collected through a confocal micro-objective setup allowing for a lateral resolution better than $1 \mu \mathrm{m}$. The continuous wave excitation was provided by solid-state lasers emitting at a wavelength of $532 \mathrm{~nm}$ and $640 \mathrm{~nm}$. The PL signal was analyzed by a grating monochromator and a silicon-based charged coupled device camera. The spectral resolution was better than $50 \mu \mathrm{eV}$ in full width at half maximum. All experiments were performed at $10 \mathrm{~K}$.

Typical PL spectra of single GaAs QDs in sample B are displayed in Fig. 1(a). Each line is attributed using various analyses including: (i) power dependence-the intensities of $\mathrm{X}$ and $\mathrm{XX}$ are well fitted by the first- and the second-order Poissonian laws, respectively, while $\mathrm{X}^{+}$and $\mathrm{X}^{-}$show an intermediate behavior, as shown in Fig. $1(\mathrm{~b}) ;{ }^{17}$ (ii) anisotropic fine structures, which governs the $\mathrm{X}$ and $\mathrm{XX}$ lines is not resolved for $\mathrm{X}^{ \pm}$as expected [Fig. 1(c)]; ${ }^{18}$ (iii) the attribution of $\mathrm{X}^{ \pm}$is not straightforward. We point out that the $\mathrm{X}^{+}$line is always present for all QDs, but the $\mathrm{X}^{-}$line is often missing, or masked by other emission signals at high excitation. Such observations, together with the fact that our samples may contain residual acceptors, led us to assign the $\mathrm{X}^{+}$line to 
(a)

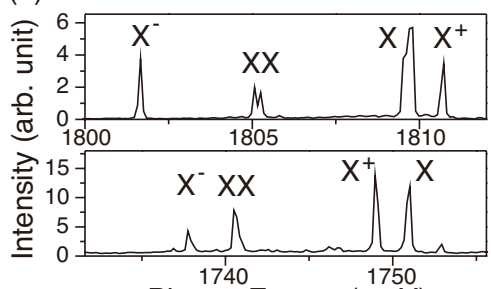

(c) Photon Energy (meV)

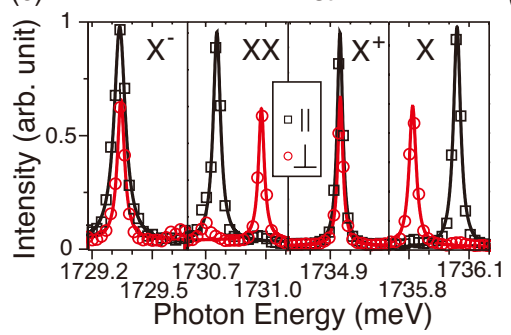

(b)

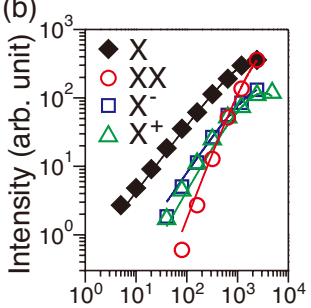

(d) Excitation Power (nW)

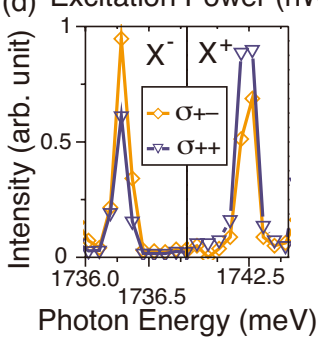

FIG. 1. (Color online) (a) PL spectra of two QDs from sample B. (b) PL intensities as a function of the incident power. Poissonian fits for $\mathrm{X}$ and $\mathrm{XX}$ and quasi-Poissonian fits for $\mathrm{X}^{ \pm}$are shown by lines. (c) Linearly polarized PL spectra, where the axis of polarization is parallel to $[110](\|)$ and $[1-10](\perp)$. (d) Circularly polarized PL spectra for $\mathrm{X}^{ \pm}$after circularly polarized excitation at a wavelength of $640 \mathrm{~nm}$. Cross-polarized and co-polarized components are denoted by $\sigma_{+-}$and $\sigma_{++}$, respectively.

positive trions. (iv) Moreover, we analyze the degree of circular polarization after circularly polarized pumping [see Fig. 1(d)]. As a result, we find that $\mathrm{X}^{+}$exhibits a significant positive degree of polarization while $\mathrm{X}^{-}$shows either negative or positive degree depending on the QD. Such signatures agree with previous works on the carrier relaxation by the electron-hole spin exchanges. ${ }^{19,20}$

A series of PL spectra for QDs with different emission energies is shown in Fig. 2. They are aligned to the $\mathrm{X}$ energy which increases from the top to the bottom panels. Every exciton complex energy systematically shifts with $\mathrm{X}$ emission energy, i.e., dot size. Most strikingly, the $\mathrm{X}^{+}$line ap-
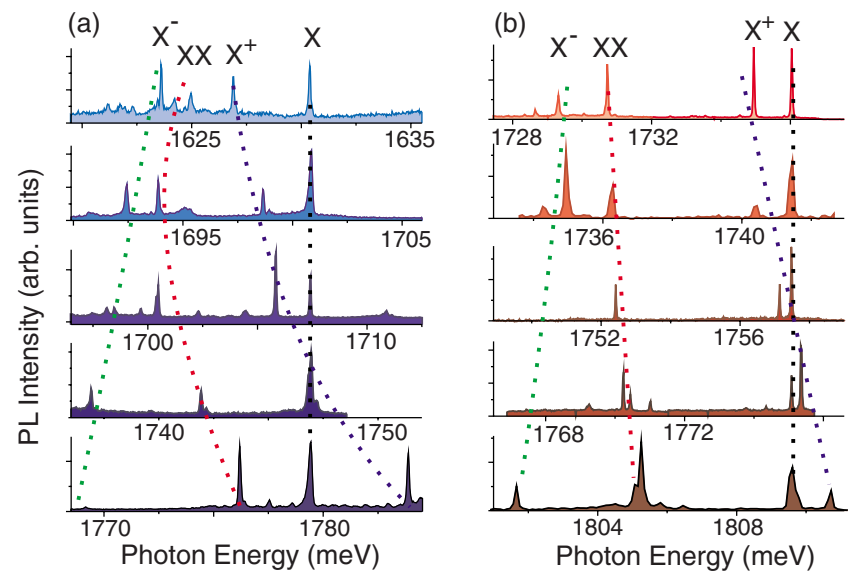

FIG. 2. (Color online) PL spectra of QDs from (a) sample A and (b) sample B. They are displayed with increasing X energy from the top to the bottom panel and are shifted to highlight the energy difference from the other lines. The dotted lines are guides to the eye.

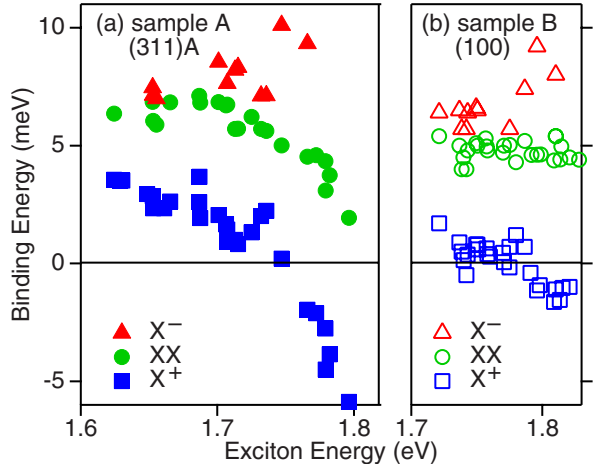

FIG. 3. (Color online) Experimental dependence of the BE of exciton complexes on the photoluminescence energy of $\mathrm{X}$ in (a) sample A and (b) sample B.

pears at the lower energy side of $\mathrm{X}$ for large QDs while it appears at the opposite side of $\mathrm{X}$ for small QDs.

The few-particle BE is experimentally defined as the spectral shift between the $\mathrm{X}$ line and the relevant exciton complex line. Figure 3 shows the evolution in the BE of each exciton complex as a function of the $\mathrm{X}$ energy (positive $\mathrm{BE}$ is measured as a red-shift with respect to the $\mathrm{X}$ line). Note that overall behaviors are qualitatively similar between sample A and sample B. Moreover, the fluctuations of the data points are remarkably small if the morphological distribution of QDs is taken into account. This finding implies that few-particle energies in strain-free systems are essentially ruled by the degree of carrier confinement, and are not strongly affected by other microscopic properties.

Pronounced features in this analysis are summarized as follows: (i) the $\mathrm{BE}$ of $\mathrm{X}^{-}$is always positive, thus $\mathrm{X}^{-}$forms a bound state. It increases from 5 to $10 \mathrm{meV}$ with increasing QD energy. (ii) $\mathrm{X}^{+}$shows a trend opposite to $\mathrm{X}^{-}$. The $\mathrm{BE}$ decreases with QD energy, from positive values to negative values, suggesting a transition from the bound state to the unbound state as QD decreases in size. This is more pronounced in sample A where the BE changes from 4 to $-6 \mathrm{meV}$. (iii) XXs shows a $\mathrm{BE}$ of about $6 \mathrm{meV}$, regardless of QD size, for large QDs in both sample A and sample B. For smaller QDs in sample A, the BE decreases to $\sim 2 \mathrm{meV}$ with QD energy, similarly to the evolution of $\mathrm{X}^{+}$.

These behaviors are understood in the following scenario. The BE of few-particle states is approximated by the sum of attractive and repulsive interactions between all pairs of particles. Such treatment, analogous to Hartree's mean-field correction, essentially reflects the difference between the wave functions of an electron and a hole, ${ }^{21}$ thus it is enhanced for smaller QDs. Since the confinement is stronger for holes than for electrons, the mean field formed by a confined exciton would act as an attractive force for a resident electron, leading to a positive $\mathrm{BE}$ for $\mathrm{X}^{-}$. The same mean field would serve as a repulsive force for a hole, thus leading to negative $\mathrm{BE}$ for $\mathrm{X}^{+}$. Consequently the same magnitude of energy renormalization, but with the opposite trends, is expected for $\mathrm{X}^{-}$and $\mathrm{X}^{+}$. This is consistent with the observations of opposite size dependences in the $\mathrm{X}^{ \pm}$energies, for QDs whose emission energy is less than $\sim 1.75 \mathrm{eV}$ in sample A. For QDs with higher energies, on the other hand, the $\mathrm{X}^{+} \mathrm{BE}$ 


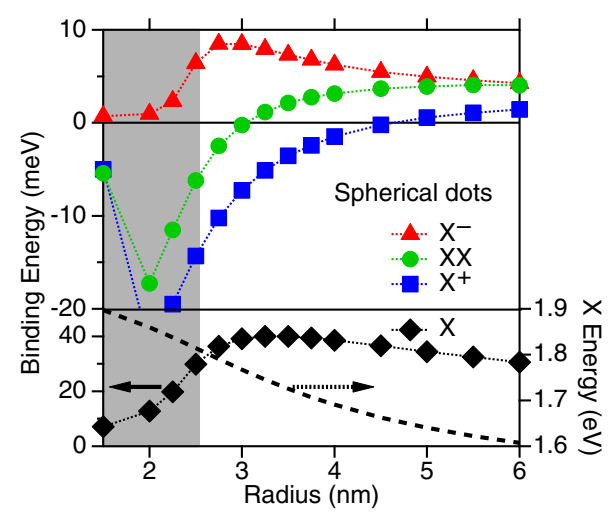

FIG. 4. (Color online) The theoretical binding energies of exciton complexes in spherical GaAs/ $\mathrm{Al}_{0.3} \mathrm{Ga}_{0.7} \mathrm{As}$ QDs as a function of radius $r$, calculated by the QMC approach. The shaded area shows a condition in which no bound state was formed for single electrons. The exciton BE and its PL energy are also plotted in the bottom panel. The parameters used are: $V_{\mathrm{e}(\mathrm{h})}=234$ (156) $\mathrm{meV}$, $m_{\mathrm{e}(\mathrm{h})} / m_{0}=0.067(0.35)$, and $\epsilon=12$, where $V_{\mathrm{e}(\mathrm{h})}$ is the electron (hole) band offset, $m_{\mathrm{e}(\mathrm{h})}$ is the electron (hole) effective mass, and $\epsilon$ is the dielectric constant.

steeply drops down due to the absence of the bound states for the electron, as will be described later.

In the mean-field treatment discussed above, separable wave functions are assumed to describe the few-particle states. The interparticle correlation results in a nonseparable dynamics, and a further energy shift to the low-energy side, i.e., toward the higher BE. ${ }^{11,22,23}$ The correlation effect becomes more effective for larger QDs since it is scaled by the ratio between the kinetic term $\left(\propto r^{-2}\right.$, where $r$ stands for characteristic QD size) and the Coulomb term $\left(\propto r^{-1}\right)$. As a result, the $\mathrm{BE}$ of $\mathrm{X}^{+}$becomes positive for weakly confined QDs as well as QWs. ${ }^{24}$ Overall features are qualitatively interpreted by relative changes in the mean-field effect and the correlation effect on the few-particle states.

We have performed QMC calculations to evaluate fewparticle correlated energies. The QMC approach enables us to determine the exact many-body ground-state energies under the effective mass approximation. The details of this method are given in Ref. 25. The BE of $\mathrm{X}^{-}$, for example, is defined as $-E_{\mathrm{X}^{-}}+E_{\mathrm{X}}+E_{\mathrm{e}}$, where $E_{\mathrm{X}^{-}}, E_{\mathrm{X}}$, and $E_{\mathrm{e}}$ are the energies of $\mathrm{X}^{-}, \mathrm{X}$, and the single-particle energy of the electron, respectively, all of which are treated on an equal footing. Note that the $\mathrm{BE}$ with this definition is equivalent to the spectral shift between the PL lines of $\mathrm{X}^{-}$and $\mathrm{X}$.

The results of QMC calculations for spherical QDs are summarized in Fig. 4. The shaded area corresponds to a condition where no bound states are formed for a single electron when $r<r_{\mathrm{c}}=\pi \hbar / \sqrt{8 m_{\mathrm{e}} V_{\mathrm{e}}} \approx 2.5 \mathrm{~nm}$, where $V_{\mathrm{e}}$ is the conduction band offset and $m_{\mathrm{e}}$ is the electron effective mass. There are indications of different kinetics involved in two regions which are separated by $r_{\mathrm{c}}$. For $r<r_{\mathrm{c}}$, every few-particle state shows reduction in its $\mathrm{BE}$ with decreasing size. This is because electrons in such a small QD are not bound by the confinement potential and are distributed outside the QD. ${ }^{26}$ Thus, the hole-hole repulsion dominates the energy renormalization in $\mathrm{X}^{+}$and $\mathrm{XX}$, pushing their $\mathrm{BE}$ to negative val-

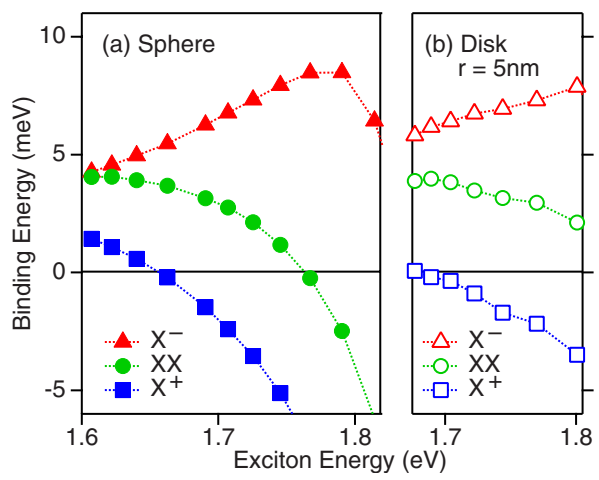

FIG. 5. (Color online) The theoretical binding energies of exciton complexes calculated as a function of exciton energy, for (a) spherical QDs and (b) disklike QDs. In the latter series, the disk radius was set to $5 \mathrm{~nm}$ and the height was varied in a range between $2 \mathrm{~nm}$ and $5 \mathrm{~nm}$ in $0.5 \mathrm{~nm}$ increments. The band parameters are the same as those used in Fig. 4.

ues, consistently with the experimental data for small QDs. Note that XX also becomes unbound for sufficiently small QDs, which is consistent with previous studies on strained QD systems. ${ }^{11}$ At the same time, the $\mathrm{X}^{-} \mathrm{BE}$ is also suppressed due to the reduction in the mean field. For $r>r_{\mathrm{c}}$, on the other hand, the size-dependence of the $\mathrm{X}^{+}$and $\mathrm{X}^{-} \mathrm{BE}$ have opposite trend and are determined by a balance of the mean field and correlation effects, as it has already been mentioned.

In order to compare these numerical results with the measured PL shifts, the few-particle BEs are plotted as a function of the $\mathrm{X}$ transition energy in Fig. 5(a). These curves agree quite well with the experimental dependence shown in Fig. 3. Furthermore, for large QDs, the mean-field contribution to the $\mathrm{X}^{ \pm}$energies depended linearly on the $\mathrm{X}$ energy. This finding is consistent with the fact that the mean field invoked by an electron's penetration outside QD is roughly proportional to its confinement energy $\left(\propto r^{-2}\right)$.

To address the impact of QD shape on the few-particle states, QMC was carried out for a disklike QD with $r$ $=5 \mathrm{~nm}$ and different heights, which were close to the actual variations in the shape of our QDs. ${ }^{27}$ Figure 5(b) shows a series of calculated BEs for the disks, which are even in quantitative agreement with the experimental evolution in Fig. 3(b). Note that the BEs of $\mathrm{X}^{+} / \mathrm{XX}$ for high-energy QDs $(\sim 1.8 \mathrm{eV})$ are significantly lower for the sphere model than for the disk model. This is readily explained by a strong exciton mean-field that formed in spheres, reflecting the carrier localization in all three dimensions. Carriers in disks, in contrast, can align laterally without penetrating outside the disk, causing a smaller mean field. It is noteworthy that analogous discussions were found in previous works on QWs, where measured trion BEs were much larger than the ones estimated theoretically, and the enhancement of $\mathrm{BE}$ arose from the lateral localization of carriers. ${ }^{6-8}$ Note also that, for interfacial GaAs QDs, ${ }^{24}$ both $\mathrm{X}^{+}$and $\mathrm{X}^{-}$showed positive BE. This is likely due to the weak lateral confinement of interfacial QDs, compared with that of dropletepitaxial QDs. In this case, the main source of the fewparticle energy shift was from the interparticle correlations, 
which led to the energy reduction in every few-particle state, and then led to positive BEs for both $\mathrm{X}^{+}$and $\mathrm{X}^{-}$.

The BEs in sample A agree with the spherical model while those in sample B agree with the disk model. This is consistent with the morphological differences between these samples. QDs in sample A, which were grown on the (311)A surface, are surrounded by the barrier material in all three directions, in the absence of a wetting layer. ${ }^{15}$ In contrast, QDs in sample B, which were grown on the (100) surface, are placed on a wetting layer with a thickness of 1-2 monolayers. Consequently, QDs in sample A exhibit the fulldirectional confinement, acting as spheres, while small QDs in sample B show relatively weak lateral confinement, acting as disks.
In conclusion, single QD PL spectroscopy on GaAs/ AlGaAs quantum dots gives precise information on Coulomb interaction among the elementary constituents of excitonic complexes. The use of strain-free QDs allows us to investigate the quantum confinement while avoiding the complications related to strain and piezoelectric field and all the other pathologies affecting Stranski-Krastanov counterparts. We find that, despite changes in shape, clear trends are found when QD decreases in size. Using the QMC approach we are able to reproduce the underlying excitonic structure and its main changes with QD size. We ascribe the observed features to a change, from weak to strong, in the confinement regime and to large differences in the localization of electron and hole wave functions.
* Present address: Laboratoire Pierre Aigrain, Ecole Normale Supérieure, 24 rue Lhomond, 75231 Paris Cedex 5, France. Marco.Abbarchi@lpa.ens.fr

†kuroda.takashi@nims.go.jp

${ }^{1}$ See, e.g., H. A. Bethe and E. E. Salpeter, Quantum Mechanics of One- and Two-Electron Atoms (Springer, Berlin, 1957).

${ }^{2}$ R. C. Miller et al., Phys. Rev. B 25, 6545 (1982); D. A. Kleinman, ibid., 28, 871 (1983).

${ }^{3}$ D. Birkedal et al., Phys. Rev. Lett. 76, 672 (1996); S. Adachi et al., Phys. Rev. B 55, 1654 (1997); W. Langbein and J. M. Hvam, ibid., 59, 15405 (1999).

${ }^{4}$ G. Finkelstein, H. Shtrikman, and I. Bar-Joseph, Phys. Rev. Lett. 74, 976 (1995); Phys. Rev. B 53, R1709 (1996).

${ }^{5}$ A. J. Shields et al., Phys. Rev. B 52, R5523 (1995); A. J. Shields et al., ibid. 52, 7841 (1995).

${ }^{6}$ C. Riva, F. M. Peeters, and K. Varga, Phys. Rev. B 61, 13873 (2000); 64, 089902(E) (2001).

${ }^{7}$ L. C. O. Dacal et al., Phys. Rev. B 65, 115325 (2002).

${ }^{8}$ A. V. Filinov et al., Phys. Rev. B 70, 035323 (2004).

${ }^{9}$ R. J. Warburton et al., Nature (London) 405, 926 (2000).

${ }^{10}$ D. V. Regelman et al., Phys. Rev. B 64, 165301 (2001).

${ }^{11}$ S. Rodt et al., Phys. Rev. B 68, 035331 (2003); S. Rodt et al., ibid. 71, 155325 (2005); A. Schliwa, M. Winkelnkemper, and D. Bimberg, ibid. 79, 075443 (2009).

${ }^{12}$ S. Kako et al., Appl. Phys. Lett. 85, 64 (2004); S. Kako et al., Nature Mater. 5, 887 (2006); D. Simeonov et al., Phys. Rev. B 77, 075306 (2008).

${ }^{13}$ F. Ding et al., Phys. Rev. Lett. 104, 067405 (2010).
${ }^{14}$ K. Watanabe et al., Jpn. J. Appl. Phys., Part 2 39, L79 (2000).

${ }^{15}$ T. Mano et al., Appl. Phys. Lett. 93, 203110 (2008).

${ }^{16}$ T. Mano et al., Nanotechnology 20, 395601 (2009).

${ }^{17}$ M. Abbarchi et al., J. Appl. Phys. 106, 053504 (2009).

${ }^{18}$ M. Abbarchi et al., Phys. Rev. B 78, 125321 (2008).

${ }^{19}$ S. Cortez et al., Phys. Rev. Lett. 89, 207401 (2002); S. Laurent et al., Phys. Rev. B 73, 235302 (2006).

${ }^{20}$ A. S. Bracker et al., Phys. Rev. Lett. 94, 047402 (2005).

${ }^{21}$ P. Lelong and G. Bastard, Solid State Commun. 98, 819 (1996).

${ }^{22}$ A. Franceschetti et al., Phys. Rev. B 60, 1819 (1999); J. Shumway, A. Franceschetti, and A. Zunger, ibid. 63, 155316 (2001); G. Bester and A. Zunger, ibid. 68, 073309 (2003).

${ }^{23}$ K. Hinzer et al., Phys. Rev. B 63, 075314 (2001).

${ }^{24}$ A. S. Bracker et al., Phys. Rev. B 72, 035332 (2005).

${ }^{25}$ T. Tsuchiya and S. Katayama, Solid-State Electron. 42, 1523 (1998); T. Tsuchiya, Physica E 7, 470 (2000); Prog. Theor. Phys. Suppl. 138, 128 (2000).

${ }^{26}$ Additional considerations about the confinement regime, based on the size-dependent diamagnetic properties, can be found in, M.-F. Tsai et al., Phys. Rev. Lett. 101, 267402 (2008); M. Abbarchi et al., Phys. Rev. B 81, 035334 (2010).

${ }^{27}$ Although we have confirmed the distribution of QD energy being governed by that of height, the mean size of actual QDs was larger than that of this model, which reproduced the experimental recombination energies. The inconsistency is likely due to the heavy- and light-hole mixing, which was ignored in this analysis. See, T. Belhadj et al., Appl. Phys. Lett. 97, 051111 (2010). 